\title{
Localized Plasmon Response Engineering in B- and N-Doped Graphene
}

Fredrik S. Hage ${ }^{1}$, Demie M. Kepaptsoglou ${ }^{1,2}$, Trevor P. Hardcastle ${ }^{3}$, Morten N. Gjerding ${ }^{4}$, Kirsten T. Winther $^{4}$, Julian A. Amani ${ }^{5}$, Hans C. Hofsaess ${ }^{5}$, Kristian S. Thygesen ${ }^{4}$ and Quentin M. Ramasse ${ }^{1,3}$

1. SuperSTEM Laboratory, SciTech Daresbury Campus, Daresbury, United Kingdom

2. Department of Physics, University of York, Heslington, York, YO10 5BR, United Kingdom

${ }^{3}$ School of Chemical and Process Engineering, University of Leeds, Leeds, LS2 9JT, United Kingdom

4. CAMD and Center for Nanostructured Graphene (CNG), Denmark Technical University, Fysikvej 1, building 307, 2800 Kgs. Lyngby, Denmark

${ }^{5}$ II Physikalisches Institut, Georg-August-Universität Göttingen, Friedrich-Hund-Platz 1, 37077

Göttingen, Germany

Due to its fascinating properties, graphene is emerging as a highly promising plasmonic material for implementation in devices aimed at applications such as non-linear optics, photo detection, light sources or even quantum optics. There is however very little information on prospects for opto-electronic graphene devices operating in the UV waveband, which would require the modification of the interband plasmon response of graphene. This could be achieved via chemical doping and the inclusion of $\mathrm{B}$ or $\mathrm{N}$ atoms in the graphene lattice is as a result the focus of extensive study in the scientific community, with the aim to modify the electronic structure of graphene. Such an approach has furthermore been predicted to induce a shift of the Fermi level resulting in $p$ or $n$ doping. In the present study, single atom $\mathrm{B}$ or $\mathrm{N}$ substitutional doping in single-layer suspended graphene was readily achieved by low energy ion implantation, using technology akin to that routinely exploited in current semi-conductor technology and therefore offering clear scaling-up prospects [1]. A $p$ and $n$ character was indeed recently demonstrated in very similar samples, using atomically-resolved scanning transmission electron microscopy (STEM) and core-loss electron energy loss spectroscopy (EELS) [2].

Here, the inclusion of single $\mathrm{B}$ and $\mathrm{N}$ dopants in the lattice is shown to induce a dampening or enhancement of the characteristic interband $\pi$ plasmon of graphene through a high-resolution electron energy loss spectroscopy study carried out on a monochromated Nion UltraSTEM100 MC capable of an energy resolution down to $14 \mathrm{meV}$. A relative $16 \%$ decrease or $20 \%$ increase in the $\pi$ plasmon quality factor is attributed to the presence of a single substitutional $\mathrm{B}$ or $\mathrm{N}$ atom dopant respectively. This modification is in both cases shown to be relatively localised, with data suggesting the plasmonic response tailoring can no longer be detected within experimental uncertainties beyond a distance of approximately $1 \mathrm{~nm}$ from the dopant [3].

Extensive $a b$ initio calculations using the local density approximation (LDA) exchange-correlation functional as implemented in the electronic structure code GPAW, confirm the trends observed experimentally. Here, we take into account local field effects, we attempt to compensate for the altered carrier concentration induced in the ground state by the presence of the substitutional defects, and we include the integration over momentum space required by the converged probe geometry. However, practical computational limitations restrict supercell sizes and prevent us from achieving the same match quality in low loss simulations as for core loss calculations [4]. Nevertheless, our results directly confirm the possibility of tailoring the plasmonic properties of graphene in the ultraviolet waveband, at the atomic scale, a crucial step in the quest for utilising graphene's properties towards the development of plasmonic and optoelectronic devices operating at ultraviolet frequencies [3,5]. 


\section{References:}

[1] U. Bangert et al., Nano Lett. 13 (2013), p. 4902-4907.

[2] D. Kepaptsoglou et al., ACS Nano 9 (2015), p.11398-11407.

[3] F.S. Hage et al., ACS Nano, Articles ASAP (2018), doi: 10.1021/acsnano.7b08650.

[4] T.P. Hardcastle et al., J. Phys.: Condens. Matter. 29 (2017), 225303.

[5] SuperSTEM is the U.K's National Research Facility for Advanced Electron Microscopy, funded by EPSRC.
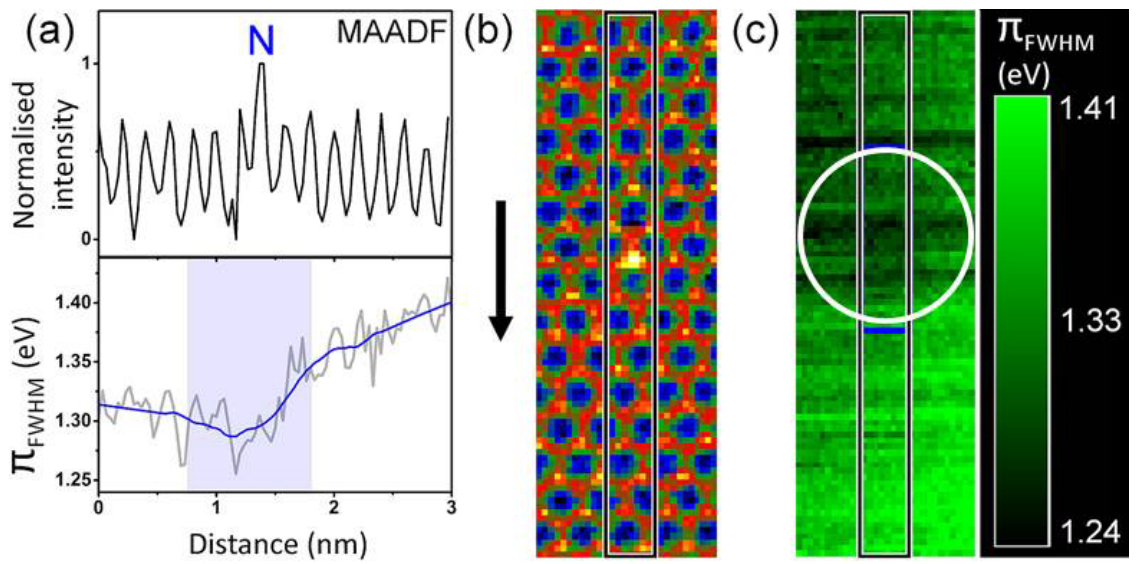

Figure 1. (a) Medium angle annular dark field (MAADF) integrated intensity line profile compared to the local change in $\pi$ plasmon peak width (FWHM) for N-doped graphene. A smoothed FWHM line profile is superimposed; (b) corresponding MAADF image; (c) $\pi$ plasmon peak FWHM maps (image width: $1.1 \mathrm{~nm}$ ). The regions from which the line profile was extracted is indicated on (c). The localisation of the observed $\pi$ plasmon enhancement $(\mathrm{N})$ is estimated to be $\sim 1 \mathrm{~nm}$, as indicated.
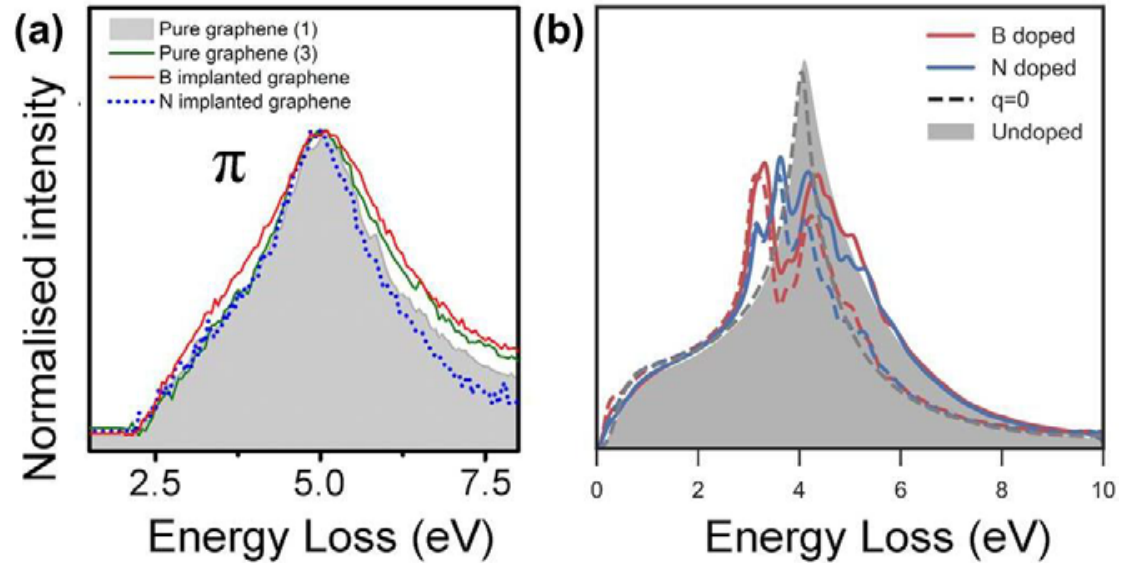

Figure 2. (a) Background-subtracted loss spectra of B and N doped graphene compared to those of the non-doped graphene patches, far away (1) or near (2) surface contamination; (b) Simulated low-loss EEL spectra comparing B-doped, N-doped and pure graphene. Full lines show the full momentumsummed EEL spectrum while the dashed lines show the response in the optical limit without local field effects. 